\title{
Revisiting the $B$-factor variation in a-SiC:H deposited by HWCVD
}

\author{
Bibhu P. Swain, Samadhan B. Patil, Alka Kumbhar, R.O. Dusane* \\ Department of Metallurgical Engineering and Materials Science, Indian Institute of Technology, Bombay, Powai, Bombay 400076, India
}

\begin{abstract}
In order to understand material properties in a better way, it is always desirable to come up with new variables that might be related to the film properties. The $B$-parameter is such a variable, which relates to the quality of a-SiC:H films both in terms of electronic and optical properties. $B$ (scaling factor) is essentially the slope of the straight-line part of the $(\alpha E)^{1 / 2}-E$ (Tauc plot). Due to dependence on a large number of parameters and no detailed research, many previous authors have surmised that $B$ has an ambiguous correlation with carbon content. We have made an attempt to establish the relation between the $B$-parameter as a quality-indicating factor of a-SiC:H films in both carbon- and silicon-rich material. For this we studied a-SiC:H films deposited by the HWCVD method with broad deposition parameters of substrate temperature $\left(T_{\mathrm{s}}\right)$, filament temperature $\left(T_{\mathrm{F}}\right)$ and $\mathrm{C}_{2} \mathrm{H}_{2}$ fraction. Our results indicate that the $B$-parameter varies considerably with process conditions such as $T_{\mathrm{F}}$, total gas pressure and carbon content. An attempt is made to correlate the $B$-parameter with an opto-electronic parameter, such as the mobility edge, which has relevance to the device-quality aspects of a-SiC:H films prepared by HWCVD.
\end{abstract}

Keywords: $\quad$ B-parameter; Hot-wire chemical vapour deposition (HWCVD); Tauc plot

\section{Introduction}

Since the first results on a-Si:H thin films prepared by hot-wire chemical vapour deposition (HWCVD), there have been various claims regarding the advantages of the HWCVD technique vis-à-vis the conventional RF glow discharge method. Issues such as hydrogen content, short- and medium-range order, Urbach energy, mobility edges and more importantly the stability of the films deposited by HWCVD are being critically analysed and the various claims are being hotly debated [1-5].

One parameter, which is closely related to the above issues, is the $B$-parameter, which appears in the Tauc formula used to determine the bandgap of the material. It has been shown that this parameter is correlated to the structural and compositional disorder through $\Delta E$, the conduction band tail width. The Mott formula, i.e. $\mathrm{B} \propto N(E)^{2} / n \Delta E$, where $n$ is the refractive index, $N(E)$ is the density of states at the band edge and $\Delta E$ is the mobility edge width, indicates the different factors that determine the value of $B$ [6].
There have been many studies on the optical properties of a-Si:H and a-SiC:H prepared by the conventional glow-discharge method [7-10]. Over the last decade the HWCVD method has been employed to deposit these thin films by various groups $[1-5,11]$. We have been successful in depositing good-quality a-SiC: $\mathrm{H}$ films that show room-temperature photoluminescence [12]. We have also been able to fabricate thin light-emitting devices out of this material. In order to understand the material properties better, we have studied the variation of the $B$-parameter in these films. In this paper we attempt to correlate variations in the $B$-parameter to the structural parameters determined from Raman spectroscopy and optical parameters determined from UV-Vis spectrophotometry data.

\section{Experimental details}

The a-SiC:H films were deposited using $\mathrm{SiH}_{4}+\mathrm{C}_{2} \mathrm{H}_{2}$ gas mixtures in a HWCVD reactor that employs a tungsten filament. The details of the hot-wire apparatus are given in an earlier publication [11]; however, for the sake of completeness the deposition parameters are given in Table 1. For the present work, samples were prepared by varying the $\mathrm{C}_{2} \mathrm{H}_{2}$ gas flow so that a-SiC:H 
Table 1

Parameters employed during a-SiC:H deposition

\begin{tabular}{lc}
\hline Gases used & $\mathrm{SiH}_{4}, \mathrm{C}_{2} \mathrm{H}_{2}$ \\
$\mathrm{C}_{2} \mathrm{H}_{2}$ fraction $\mathrm{C}_{2} \mathrm{H}_{2} /\left[\mathrm{C}_{2} \mathrm{H}_{2}+\mathrm{SiH}_{4}\right]$ & $0-0.83$ \\
Substrate temperature $\left(T_{\mathrm{s}}\right)$ & $250{ }^{\circ} \mathrm{C}$ \\
Filament temperature $\left(T_{\mathrm{F}}\right)$ & $1700{ }^{\circ} \mathrm{C}$ \\
Total gas pressure & $100 \mathrm{mTorr}$ \\
Filament-substrate distance & $5 \mathrm{~cm}$ \\
\hline
\end{tabular}

films were obtained with band gap varying from 1.75 $\mathrm{eV}$ for $\mathrm{C}_{2} \mathrm{H}_{2}$ at $0 \mathrm{sccm}$ to $3.4 \mathrm{eV}$ for $\mathrm{C}_{2} \mathrm{H}_{2}$ at $10 \mathrm{sccm}$. The $\mathrm{SiH}_{4}$ flow was kept constant at $2 \mathrm{sccm}$. The bandgap of films deposited on Corning 7059 glass was determined from the optical transmission spectra. In addition, Raman scattering measurements were performed on some typical samples to determine the structural order [11].

\section{Results and discussion}

Fig. 1 shows the Tauc plot for HWCVD a-SiC:H films deposited with different $\mathrm{C}_{2} \mathrm{H}_{2}$ fractions in the gas phase. In a $(\alpha E)^{1 / 2}-E$ plot, the intercept of the straight line with the $E$ axis gives the Tauc bandgap $\left(E_{\text {Tauc }}\right)$, while the slope of the line yields the $B$-parameter. It is clear that $E_{\text {Tauc }}$ increases with increasing $\mathrm{C}_{2} \mathrm{H}_{2}$ fraction and a maximum of $3.2 \mathrm{eV}$ is obtained for $\mathrm{C}_{2} \mathrm{H}_{2} /\left(\mathrm{C}_{2} \mathrm{H}_{2}+\right.$ $\left.\mathrm{SiH}_{4}\right)=0.83$. We simultaneously determined the bandgap $E_{04}$ for the films from the plot of the absorption coefficient, $\alpha$, vs. wavelength obtained from UV-Vis measurements. $E_{04}$ corresponds to the energy at which the absorption coefficient has a value of $10^{4} \mathrm{~cm}^{-1}$ $[10,13]$. The difference between the $E_{04}$ and $E_{\text {Tauc }}$, i.e. $\left(E_{04}-E_{\text {Tauc }}\right)$ is plotted as a function of $E_{\text {Tauc }}$ in Fig. 2.

From Fig. 2 it is evident that as the bandgap $E_{\text {Tauc }}$

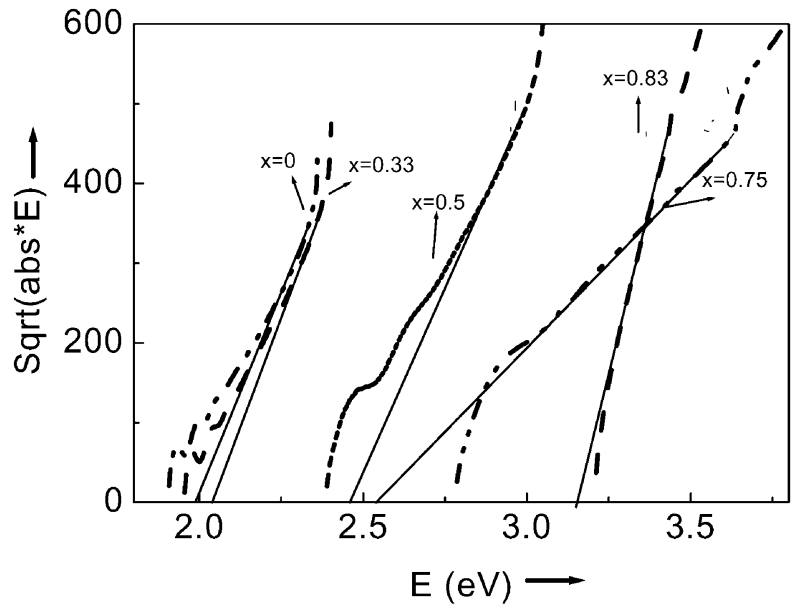

Fig. 1. Tauc plot of a-SiC:H films deposited with different $\mathrm{C}_{2} \mathrm{H}_{2} /\left(\mathrm{C}_{2} \mathrm{H}_{2}+\mathrm{SiH}_{4}\right)$ ratios at $T_{\mathrm{f}}=1700{ }^{\circ} \mathrm{C}$ and $T_{\mathrm{s}}=250{ }^{\circ} \mathrm{C}$. Dashed lines are the Tauc plot, and the intercept of the linear fit line with the energy axis indicates the Tauc bandgap.

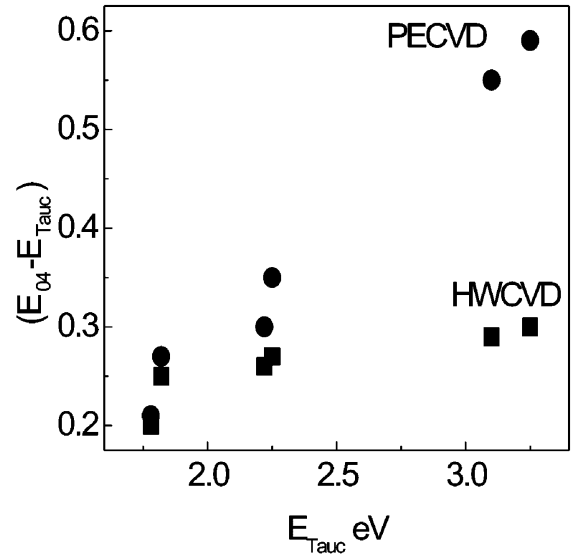

Fig. 2. Variation of difference in the bandgap determined from the two methods, i.e. $\left(E_{04}-E_{\text {Tauc }}\right)$, as a function of $\mathrm{C}_{2} \mathrm{H}_{2}$ fraction for HWCVD and PECVD a-SiC:H films deposited at $T_{\mathrm{F}}=1700{ }^{\circ} \mathrm{C}$ and $T_{\mathrm{s}}=250{ }^{\circ} \mathrm{C}$

increases, the difference $\left(E_{04}-E_{\text {Tauc }}\right)$ also increases up to $E_{\text {Tauc }}=3.0 \mathrm{eV}$ and tends to saturate beyond this value. Also shown in the figure are data points obtained for the glow discharge material $[8,9]$. We attempt to interpret these results on the basis of some earlier observations on glow discharge a-SiC:H material [13-17], which indicate an increase in the width of conduction band mobility edges as the carbon content in the film increases. What is interesting in the present case is that $\left(E_{04}-E_{\text {Tauc }}\right)$ saturates at a much lower value of $\sim 0.3$ $\mathrm{eV}$ compared to that for the glow discharge material [9]. Whether this is related to a different film growth mechanism in the HWCVD or the $\mathrm{C}_{2} \mathrm{H}_{2}$ precursor cannot be conjectured on the basis of the present data.

Fig. 3 shows the variation of $B(\mathrm{eV} \mathrm{cm})^{-1 / 2}$, i.e. the slope of the Tauc plot, for different $\mathrm{C}_{2} \mathrm{H}_{2}$ fractions. It is evident that $B$ is highest for $\mathrm{C}_{2} \mathrm{H}_{2}$ at $0 \mathrm{sccm}$ and decreases with increasing carbon content in the film.

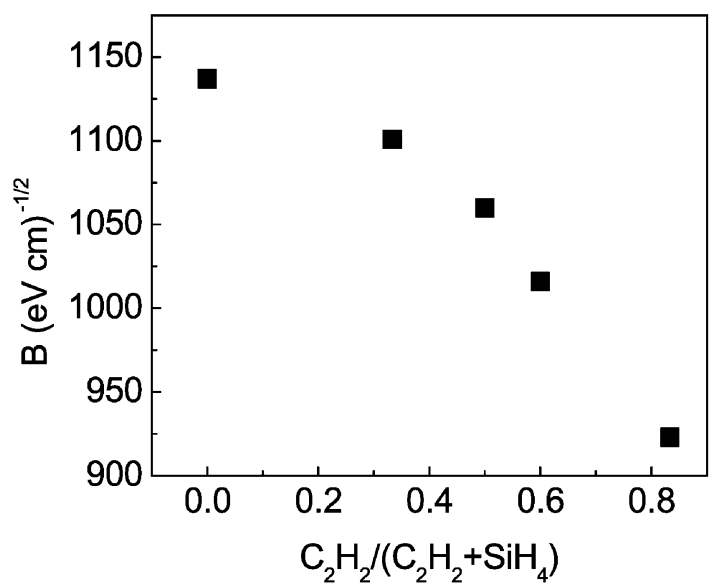

Fig. 3. Variation of $B$-parameter with $\mathrm{C}_{2} \mathrm{H}_{2}$ fraction for HWCVD aSiC: $\mathrm{H}$ films deposited at $T_{\mathrm{F}}=1700{ }^{\circ} \mathrm{C}$ and $T_{\mathrm{s}}=250{ }^{\circ} \mathrm{C}$. 


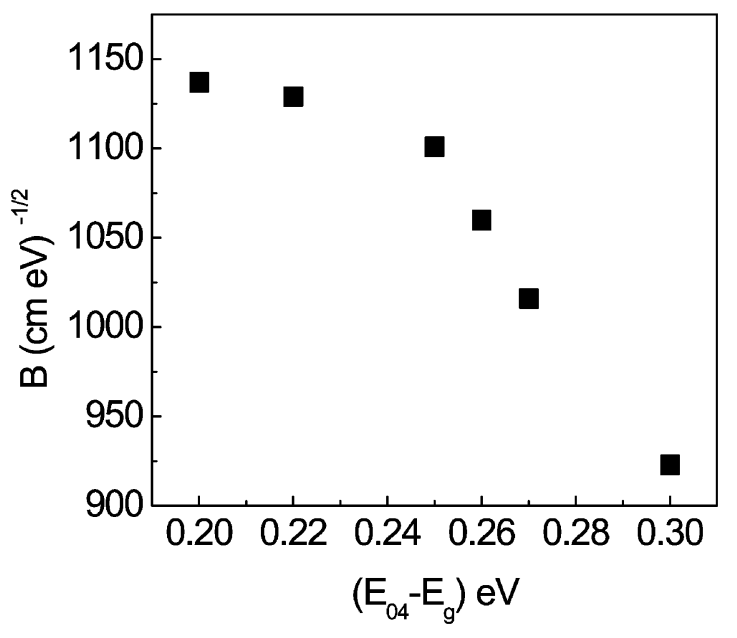

Fig. 4. Correlation between the $B$-parameter and $\left(E_{04}-E_{\text {Tauc }}\right)$ for the hot-wire a-SiC: $\mathrm{H}$ films deposited with increasing $\mathrm{C}_{2} \mathrm{H}_{2}$ gas fraction at $T_{\mathrm{s}}=250{ }^{\circ} \mathrm{C}$ and $T_{\mathrm{F}}=1700{ }^{\circ} \mathrm{C}$.

There have been many attempts to attribute the $B$ parameter to different aspects of the network in a-Si:H. Such attempts are mainly through correlation of the $B$ parameter with the width of the conduction band mobility edge [16]. This is in turn related to deviations in network bonding [18]. There has been yet another attempt to correlate the $B$-parameter to the difference between $E_{04}$ and $E_{\text {Tauc }}$ (bandgap energy calculated from the Tauc plot) We show such a correlation in Fig. 4, which reflects variation of the $B$-parameter as a function of $\left(E_{04}-E_{\text {Tauc }}\right)$. It is evident that $B$ decreases with increasing $\left(E_{04}-E_{\text {Tauc }}\right)$. In the case of GD-deposited material, $\left(E_{04}-E_{\text {Tauc }}\right)$ increases linearly with increasing carbon content, while it saturates at approximately 0.3 for the HWCVD material.

In the present work we make a further attempt to correlate $B$ to the structural aspect of bond angle deviation. The information about bond angle deviation $\Gamma=15+6 \Delta \theta_{\mathrm{b}}$ [19] can be determined from the width of the TO peak in the Raman spectrum, denoted as Гто $[18,20]$. However, in the present work, we make only qualitative observations as shown in Fig. 5, where we have depicted the Raman spectra of a Si:H (curve A) and a-SiC:H (curve B) thin films deposited by the HWCVD method. $\Gamma_{\text {то }}$ for a-SiC:H is significantly larger compared to that for a-SiH, indicating an increase in the bond angle deviation. Thus, at least qualitatively, it can be said that carbon incorporation in a-Si:H leads to an increase in structural disorder in terms of bond angle, which has been observed earlier [20]. This, in turn, could be correlated with measured $\left(E_{04}-E_{\text {Tauc }}\right)$ and the $B$-parameter.

\section{Conclusion}

We have reported the results of the first attempts to analyse the optical characteristics of HWCVD a-SiC:H

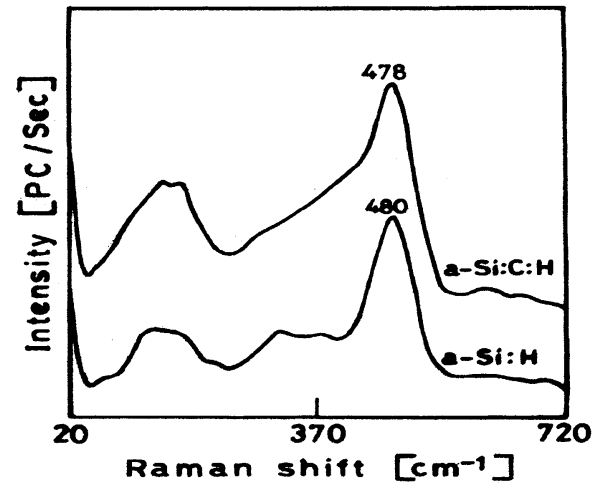

Fig. 5. Raman spectra for two typical films deposited by HWCVD: (a) hot wire-deposited a-SiC:H with $\mathrm{C}_{2} \mathrm{H}_{2}$ fraction of 0.33 ; and (b) intrinsic a-Si:H films.

thin films prepared using a $\mathrm{SiH}_{4}+\mathrm{C}_{2} \mathrm{H}_{2}$ gas mixture. Qualitatively, the results indicate that the HWCVD a-SiC:H material behaves in a way similar to glow discharge material, with some indications of better structural order in the large-bandgap films. This is suggested by the lower $\left(E_{04}-E_{\text {Tauc }}\right)$ and higher $B$-factor for these films. Further detailed work is needed to reach a quantitative conclusion on correlating the $B$-parameter to structural disorder in the material.

\section{References}

[1] H. Matsumura, Thin Solid Films 395 (2001) 1.

[2] A.H. Mahan, Thin Solid Films 395 (2001) 12.

[3] R.E.I. Schropp, Thin Solid Films 395 (2001) 17.

[4] A. Gallagher, Thin Solid Films 395 (2001) 25.

[5] A. Ledermann, U. Weber, C. Mukherjee, B. Schroeder, Thin Solid Films 395 (2001) 61.

[6] N.F. Mott, E.A. Davis, Electronic Process in Non-Crystalline Materials, Clarendon Press, Oxford, 1979, p. 289.

[7] W.B. Jackson, S.M. Kelso, C.C Tsai, J.W. Allen, S.J. Oh, Phys Rev. B 31 (1985) 5187.

[8] D.K. Basa, Thin Solid Films 406 (2002) 75.

[9] R. Tsu, P. Menna, A.H. Mahan, Sol. Cells 21 (1987) 189.

[10] N. Saito, N. Tanaka, I. Nakaki, Appl. Phys. A 38 (1985) 37.

[11] A.A. Kumbhar, R.O. Dusane, S. Bauer, B. Schroeder, J. NonCryst. Solids 227-230 (1998) 452.

[12] A. Kumbar, S.B. Patil, S. Kumar, R. Lal, R.O. Dusane, Thin Solid Films 395 (2001) 244.

[13] D. Della Sala, P. Fiorini, A. Vrova, A. Gregori, J. Non-Cryst. Solids 77/78 (1985) 853.

[14] R. Meaudre, A. Mezhoudi, Philos. Mag. B 50 (1984) L63.

[15] A.R. Zanatta, I. Chambouleyron, Phys. Rev. B 53 (1996) 3833.

[16] N. Saito, T. Yamada, T. Yamaguchi, Philos. Mag. B 52 (1985) 987.

[17] V. Chu, J.P. Conde, J. Jarego, P. Brogueira, J. Rodriguez, N. Barradas, J.C. Soares, J. Appl. Phys. 78 (1995) 3165.

[18] Y. Hishikawa, K. Watanabe, S. Tsuda, M. Ohnishi, K. Yukinori, Jpn. J. Appl. Phys. 24 (1985) 385.

[19] D. Beeman, R. Tsu, M.F. Thorpe, Phys. Rev. B 32 (1985) 874.

[20] A. Morimoto, S. Oozora, M. Kumeda, T. Shimuzu, Solid-State Commun. 47 (1983) 773. 